\title{
Nivel de conocimiento de bioseguridad asociado a accidentabilidad laboral en personal asistencial de EsSALUD
}

\section{Level of knowledge of biosecurity associated with occupational accidents in assistant personnel of EsSALUD}

\author{
Cesar Reyes Luján ${ }^{1 *}$, Iris Carrasco Diaz ${ }^{2}$ \\ ${ }^{1}$ Universidad Nacional del Centro del Perú, ${ }^{2}$ Universidad Continental
}

\section{RESUMEN}

El objetivo fue determinar los diferentes niveles de conocimiento de bioseguridad que influyen en la accidentabilidad laboral del personal asistencial CAP II CHILCA EsSALUD 2015. El estudio fue de diseño descriptivo, correlacional, prospectivo de corte transversal; efectuado a la población de 30 trabajadores asistenciales que laboran en el Centro de Atención Primaria; para la recolección de datos se utilizó un cuestionario, y se contrastó la hipótesis mediante la prueba de Independencia estadística distribución de Chi cuadrado. Se tiene como resultados que, el 56,7 \% del personal evaluado tiene un conocimiento bajo de bioseguridad; el $20 \%$ del personal presentaron accidentes laborales. Existe una relación significativa $(p<0,05)$ entre el nivel de conocimientos de bioseguridad y la accidentabilidad laboral según edad de 41-50 años, ocupación en el grupo de vigilantes, limpieza y de enfermeras; entre aquellos que no conocen principios de universalidad, uso de barreras; escala de riesgos y también existe relación significativa entre la respuesta incorrecta sobre el riesgo biológico. En conclusión, el nivel de conocimientos de bioseguridad y la accidentabilidad laboral en los trabajadores no fue significativa $(p>0,05)$, sin embargo se observa que el $3,3 \%$ de trabajadores con nivel de conocimiento alto no tuvo accidente laboral, mientras que los de nivel de conocimiento mediano y bajo el $20 \%$ tuvo accidentes laborales; lo que nos indica que si mejoráramos el nivel de conocimiento de bioseguridad la incidencia de accidentabilidad sería menor.

Palabras clave: Bioseguridad, accidentes laborales, nivel de conocimiento.

\begin{abstract}
The objective was to determine the different levels of knowledge of biosecurity that influence the work accidents of the health care personnel CAP II CHILCA EsSALUD 2015. The study was of descriptive, correlational, prospective cross-sectional design, made to the population of 30 health workers who work in the Primary Care Center; a questionari was used for data collection, and the hypothesis was tested by means of the test for the statistical Independence of Chi square distribution. The results show that, $56,7 \%$ of the personal evaluated have a low knowledge of biosafety; $20 \%$ of the personnel presented work accidents. There is a significant relationship $(p<0,05)$ between the level of knowledge of biosafety and the work accidents rate according to age of 41-50 years, occupation in the group of security guards, cleaning and nurses; among those who do not know principles of universality, use of barriers; risk scale and there is also a significant relationship between the incorrect response on biological risk. In conclusión, the level of knowledge of biosecurity and the labor accidentability in workers was not significant $(p>0,05)$, however, it is observed that $3,3 \%$ of workers with a high level of knowledge did not have an accident at work, while those with a médium level of knowledge and under $20 \%$ had work accidents; this indicates that if we improve the level of knowledge of biosecurity the incidence of accidents would be lower.
\end{abstract}

Keywords: Biosafety, occupationals accidents, level of knowledge.

Historial del artículo:

Recibido, 04 de marzo 2016; aceptado, 10 de mayo de 2016; disponible en línea, 15 de julio de 2016

* Docente de la Facultad de Medicina Humana de la Universidad Nacional del Centro del Perú.

Correo: cesarlujan77@hotmail.com 


\section{INTRODUCCIÓN}

Los accidentes laborales constituyen un problema importante a nivel de salud, éste genera pérdidas no solo económicas, sino también sociales, laborales, legales, tanto para el trabajador como para las instituciones sanitarias (Telles, 2008).

En la última década el número de lesiones y enfermedades ocupacionales ha ido en aumento, se sabe, que, según la OMS, en el 2007, reportó a nivel mundial a 2 millones de Trabajadores del Sector Salud (TSS) que experimentaron accidentes laborales por exposición percutánea a enfermedades infecciosas anualmente, además, éste, es uno de los mecanismos de producción seguido, del contacto cutáneo mucoso y contagio por vía respiratoria; el integrante a quien se le atribuye este problema es al personal de Enfermería por pasar más tiempo con el enfermo(60-70\%) (Córdova, 2007).

Las enfermedades infecciosas tienen mayor relevancia para el personal de salud que para cualquier otra categoría profesional, ya que su práctica involucra una alta manipulación de elementos corto punzantes, así como el manejo de líquidos orgánicos potencialmente infecciosos que pueden representar un riesgo para la salud (Sedano, 2007).

Las principales enfermedades infecciosas al que está expuesto el personal de salud son: Hepatitis B (HVB), hepatitis $\mathrm{C}(\mathrm{HVC})$, Tuberculosis (TBC) y VIH, en donde, las probabilidades de desarrollar infección después de un contacto con fuente positiva son para HVB (30\%), HVC (1,8\%), VIH exposición percutánea $(0,3 \%), V I H$ exposición mucosa (0,09\%) (Miranda, 2007).

De la misma forma se sabe que el riesgo de contagio del personal de enfermería en la accidentabilidad laboral por punción con aguja o instrumento contaminado con sangre infectada es del 0,03\%, porcentaje mínimo que debe ser tomado en cuenta (Paucar, 2007).

Chile realizó un estudio donde se determinó un alto número de exposiciones a agentes biológicos por parte del personal de enfermería; el cual dio como resultado 2689 casos de enfermeras(o) expuestos a este tipo de agente infeccioso (Ramos, 2007).

Una causa importante para que se produzca este problema es el incumplimiento de normas de trabajo seguro, ligados a un nivel de conocimientos de bioseguridad. Alrededor del $90 \%$ de las exposiciones ocupacionales que originan la accidentabilidad laboral ocurren en países en desarrollo (Gambino, 2011).

En el ámbito europeo las cifras de accidentabilidad laboral son altas con mecanismos de producción antes mencionados; además un 2,8 \% de los estudiantes de
Enfermería, ya representan resultados positivos a uno de los marcadores de la HVB durante su etapa de formación (Ministerio de Ciencia, Tecnología y Medio Ambiente, 2011).

La generación de un accidente laboral tiene relación estrecha con los elementos básicos de bioseguridad, por las propias infraestructuras de los hospitalesdiseño y construcción de la instalación-. A pesar de que los profesionales de enfermería, conocen como evitar las exposiciones a agentes biológicos no son puestos en práctica los métodos de barrera como son: guantes, gorro, mascarillas, bata, lentes protectores favoreciendo el aumento de los accidentes laborales.

En EE.UU. se estima que el personal hospitalario tiene una probabilidad de un $40 \%$ a perder horas de trabajo por una enfermedad ocupacional que el resto de la PEA (Jahsi, 2011).

Latinoamérica tiene una prevalencia de enfermedades profesionales en la transmisión, mayormente, de HVB (52\%) (Romero, 2007).

Perú es uno de los países a nivel mundial que no es ajeno de estas cifras, hay una mayor proporción de que accidentes laborales ocurran en el sexo femenino, superando el 70 \% (Ramos, 2006).

ESSALUD publica en el 2003 un estudio en el que los accidentes laborales son mayores (52 \%) en comparación al año 2000 , donde la cifra es mucho menor (17\%) (Galván, 2006).

La prevención es un escalón importante para tratar los problemas en salud, diversos países ya han implementado programas de prevención de infecciones y manejo de riesgos laborales como el Caribe, Venezuela, Sudáfrica, Vietnam, asimismo el personal de enfermería debe tomar conciencia de su nivel de conocimientos de bioseguridad para evitar problemas en salud; se debe diseñar una adecuada vigilancia epidemiológica al igual que una regulación y control del reporte obligatorio de accidentes de trabajo, mejorar las políticas de educación sanitaria en salud ocupacional a nivel nacional, regional, local, cumpliendo metas a corto y largo plazo en cada hospital.

La presente investigación se justifica y es de gran importancia porque:

a) La notificación de accidentes laborales en los establecimientos de salud es actualmente un indicador obligatorio en la Instituciones como Es salud que nos permite conocer la eficiencia organizacional y su adecuación con la bioseguridad Este trabajo de investigación contribuirá a determinar la Asociación entre el nivel 
de conocimiento de Bioseguridad y la notificación de accidentes de trabajo y luego de ello posibilitar planes de mejora para disminuir esta incidencia.

b) Desde el punto de vista económico este estudio podrá plantear estrategias para disminuir la incidencia de accidentes laborales, y por ende de disminuir los costos en el diagnóstico y tratamiento de recursos humanos reflejada en la disminución de horas programas por ausencia laboral y la disminución que hace que las metas no puedan cumplirse.

c) Desde el punto de vista social este estudio contribuirá a que los actores sociales comprometidos en el presente estudio puedan valorar la implicancia de su no capacitación en el aumento de la incidencia de accidentes de trabajo.

El objetivo general de este trabajo de investigación fue de determinar los diferentes Niveles de Conocimientos de Bioseguridad que influyen en la accidentabilidad laboral en el Personal Asistencial del CAP II Chilca Es salud 2015. Los Objetivos Específicos fueron: Relacionar el nivel de conocimientos de Bioseguridad y la Accidentabilidad Laboral según sexo, edad, tiempo de trabajo, especialidad, condición laboral, estado civil, integrante de un comité y última capacitación del Personal Asistencial del CAP II Chilca Es salud 2015; verificar el nivel de conocimientos acerca de los Principios Básicos de Bioseguridad en la influencia de la accidentabilidad laboral del Personal Asistencial del CAP II Chilca Es salud 2015; evaluar el Nivel de Conocimientos de Bioseguridad en relación a la Accidentabilidad Laboral según Escala de Riesgos y Riesgo Biológico en el Personal Asistencial del CAP II Chilca ES Salud 2015; y evaluar las características de riesgo de causas y consecuencias del personal asistencial que tuvieron accidentes laborales en el CAP II Chilca Es salud 2015.

\section{MATERIAL Y MÉTODOS}

El tipo de investigación es básica porque lleva a la búsqueda de nuevos conocimientos y campos de investigación sobre Bioseguridad.

La población estuvo conformada por todo el personal asistencial que actualmente labora en el CAP II Chilca ES salud 2015, entre enfermeras, técnicos, médicos, odontólogos, obste trices, otros y personal de limpieza y administrativos. El tipo de muestreo fue no probabilística por conveniencia, el tamaño de muestra fue equivalente a la población haciendo un total de 30 trabajadores.

El alcance fue descriptivo correlacional porque se analiza e interpreta los hechos ó fenómenos relacionados al nivel de conocimientos sobre Bioseguridad y accidentabilidad laboral.

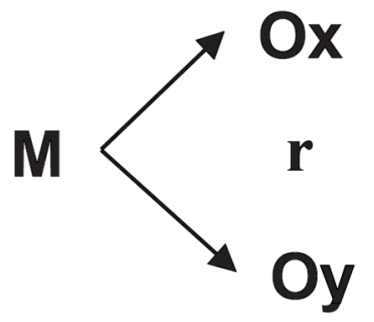

El diseño fue no experimental transversal descriptivo correlacional, que se diagrama de la siguiente manera: Donde:

M : Personal asistencial del CAP II Chilca Es salud

Ox: Niveles de Conocimientos de Bioseguridad.

Oy: Accidentabilidad Laboral.

$r \quad$ : Es la correlación de las dos variables

Se trató de buscar la relación que existe entre Nivel de Conocimientos de Bioseguridad y la Accidentabilidad Laboral en el Personal asistencial del CAP II Chilca Es salud 2015; de corte transversal ya que nos permitió obtener información acerca de las variables de estudio en un tiempo de espacio determinado en su ambiente laboral, y es prospectivo ya que los hechos se registraron a medida que ocurrieron.

La técnica de recolección de datos fue la encuesta, la misma que facilitó la participación del personal asistencial del CAP II Chilca ESSALUD 2015 para obtener la información necesaria.

El instrumento de recolección de datos fue un ccuestionario que permitió exponer y recopilar los objetivos, instrucciones, datos generales (Edad, sexo, estado civil, tiempo en que se encuentra laborando, especialidad, otros) y las preguntas necesarias, de acuerdo a los objetivos que se quiere alcanzar, haciendo un total de 21 ítems; preguntas cerradas politómicas, en las que se presenta 4 capítulos: Información general, conocimientos sobre bioseguridad (definiciones, principios, elementos básicos, medidas de eliminación, entre otros), accidentes laborales (ocurrencia, actividades preventivas y al realizarse después del accidente), enfermedades infectocontagiosas (enfermedad por accidente laboral: HVB, HVC, VIH, TBC), finalizando con un espacio para sugerencias por parte del participante.

El procesamiento de los datos se realizó de manera automatizada utilizando el software estadístico Microsoft Excel y el Programa estadístico SPSS versión 23.0. Se hizo uso de la estadística descriptiva, mediante tablas de frecuencia, tablas de doble y triple entrada con única y doble matriz, éstos, con su respectiva significancia estadística, esta información, complementada con gráficos de barras, diagrama circular y para encontrar asociación entre variables 
y contrastar las hipótesis se realizó la Prueba de Independencia Estadística de X2 (Chi cuadrado) con un nivel de significación $\alpha=0,05$, al $95 \%$ de nivel de confianza.

El presente trabajo de investigación se desarrolló en el Centro de Atención Primaria (CAP) II Chilca - Es Salud, situado en Av. Real No.1183 en el distrito de Chica de la provincia de Huancayo, región Junín en Perú.

\section{RESULTADOS}

Para el presente estudio se encuentra los siguientes

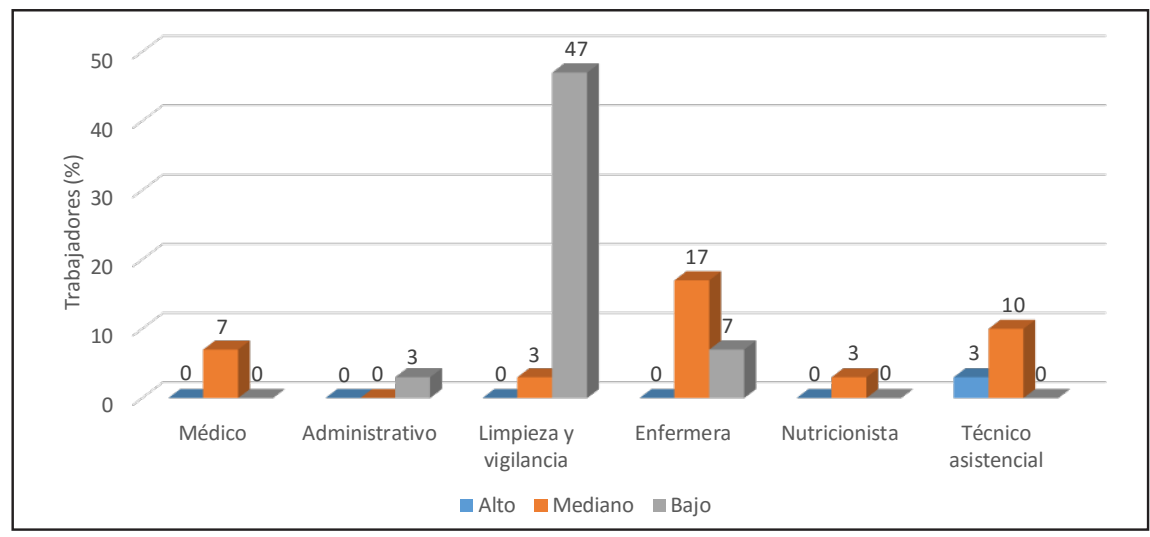

Figura 1: Nivel de conocimiento de bioseguridad según ocupación en el CAP.II Chilca-EsSalud-Huancayo 2015.

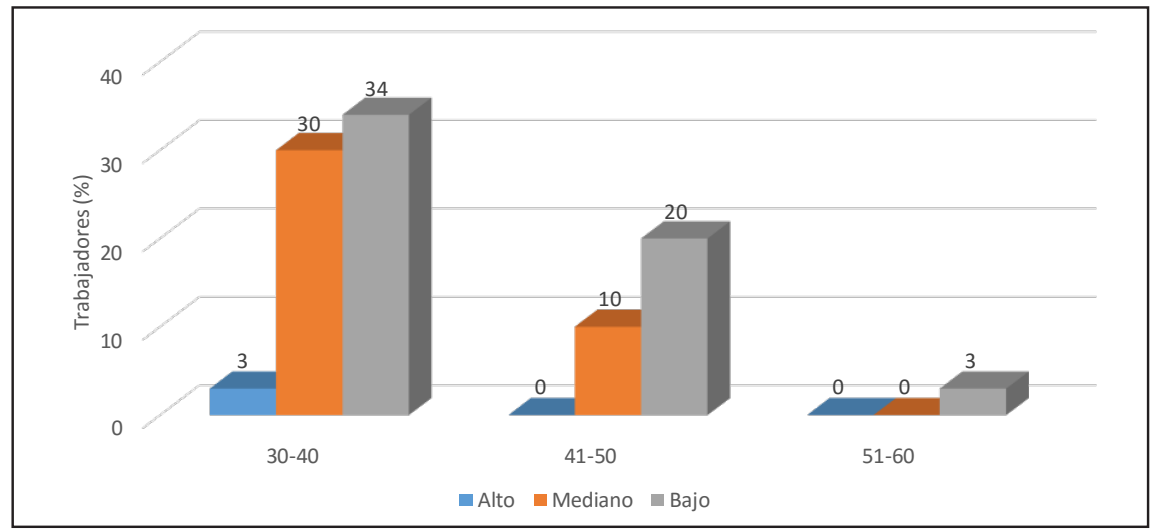

Figura 2: Nivel de conocimiento de bioseguridad según edad en el CAP.ll ChilcaEsSalud-Huancayo 2015.

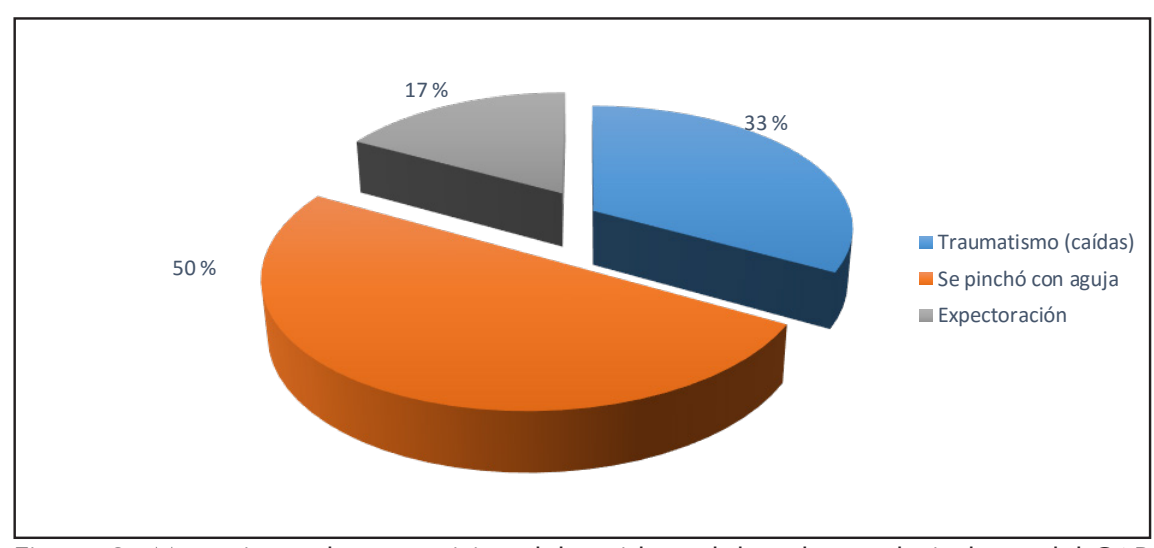

Figura 3: Mecanismo de transmisión del accidente laboral en trabajadores del CAP. II Chilca-EsSalud-Huancayo 2015. 


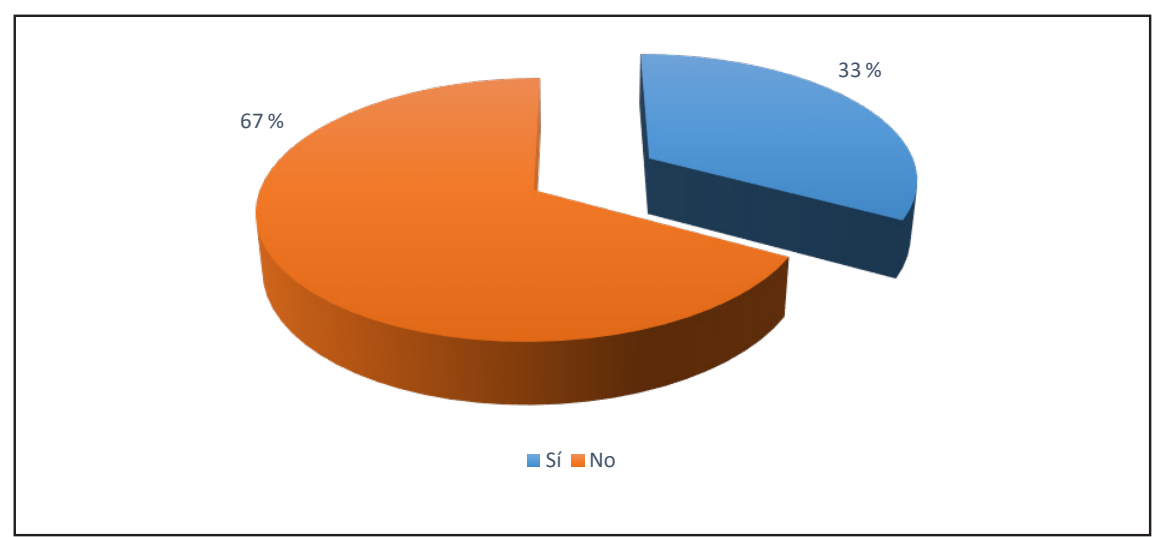

Figura 4: Elemento protector de bioseguridad al momento del accidente en trabajadores del CAP.II Chilca-EsSalud-Huancayo 2015.

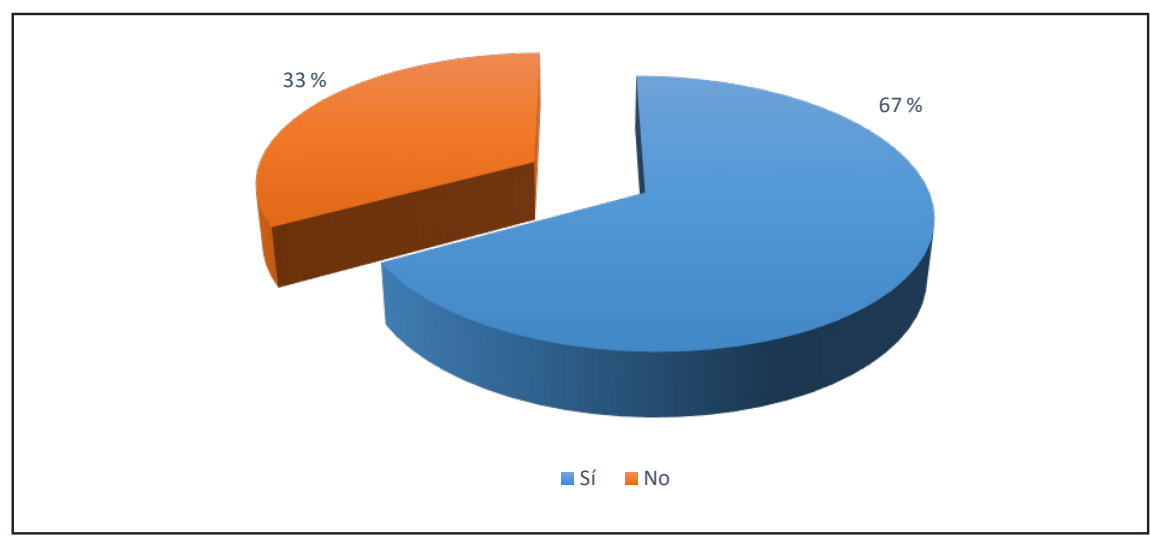

Figura 5: Inmunización activa al momento del accidente en trabajadores del CAP.II Chilca-EsSalud-Huancayo 2015.

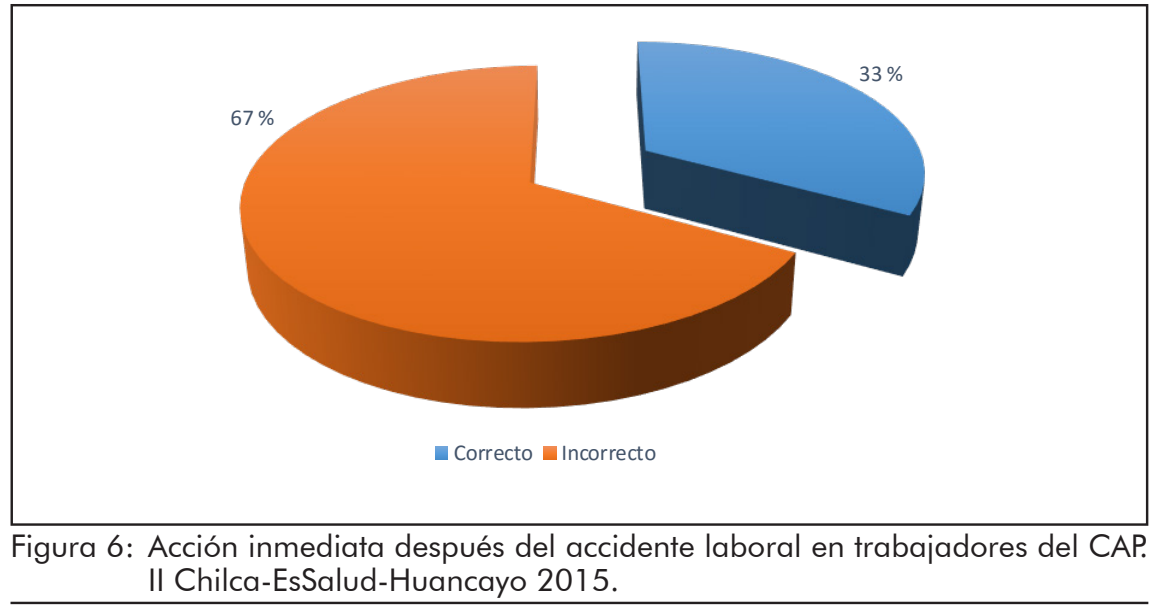

resultados:

De acuerdo a la figura 1 se muestra que el nivel de conocimiento de bioseguridad es bajo en el personal de vigilancia y limpieza (47 \%), administrativos (3\%) y enfermera (7\%).
De acuerdo a la figura 2 se muestra que el nivel de conocimiento de bioseguridad es bajo en las edades de $30-40$ años (34 \%) y de $41-50$ años (20\%) con $3 \%$ los de 51-60 años.

La figura 3 muestra que el $50 \%$ de accidentes se suscita por el pinchazo de agujas, el $33 \%$ por 


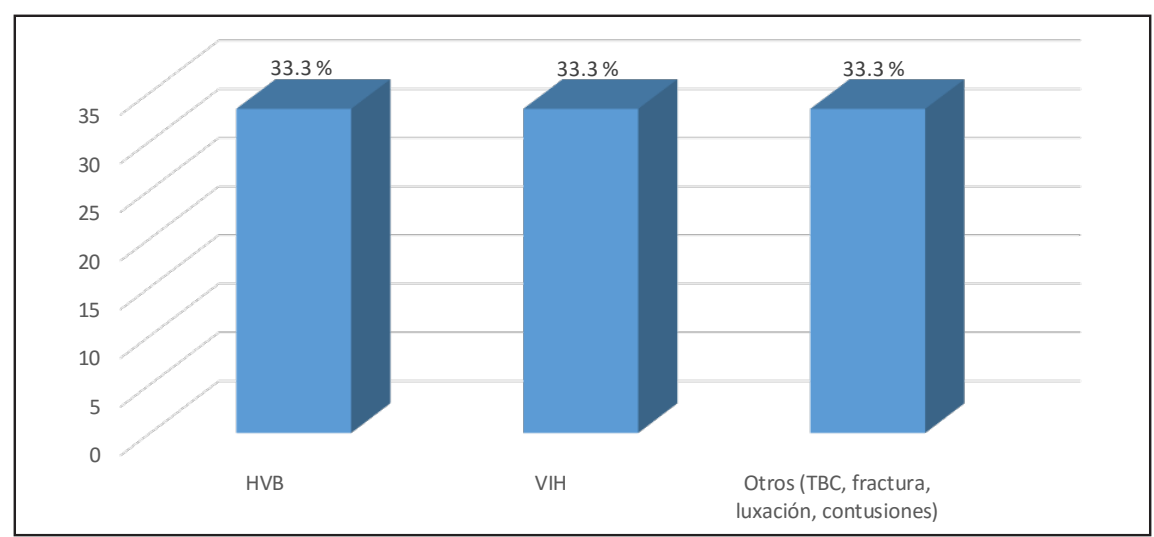

Figura 7: Tipo de enfermedad que creen estar contagiados por accidente laboral los trabajadores del CAP.II Chilca-EsSalud-Huancayo 2015.

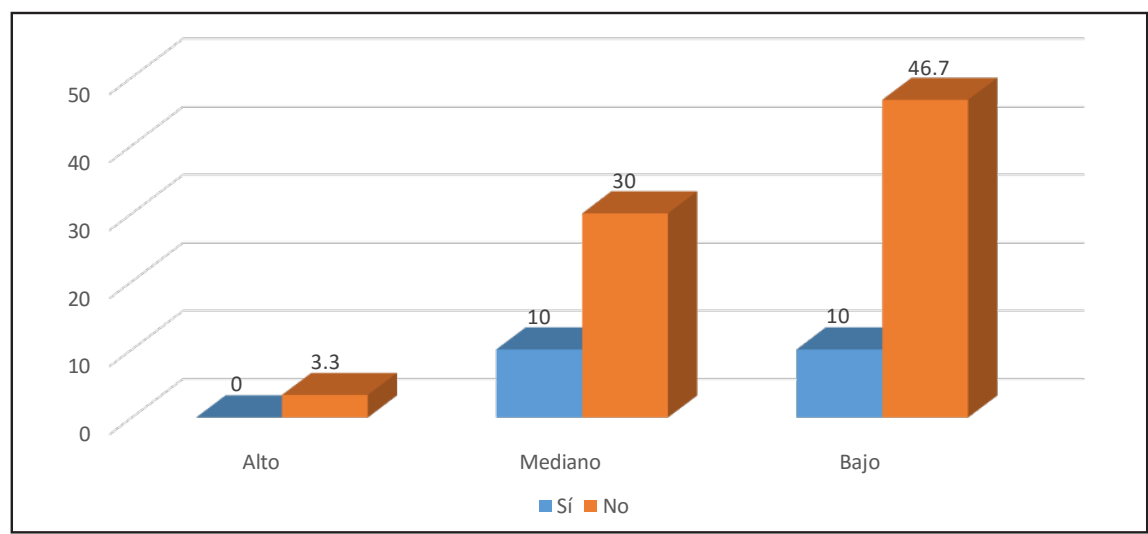

Figura 8: Relación entre el nivel de conocimiento de bioseguridad y los accidentes laborales de los trabajadores del CAP.II Chilca-EsSalud-Huancayo 2015.

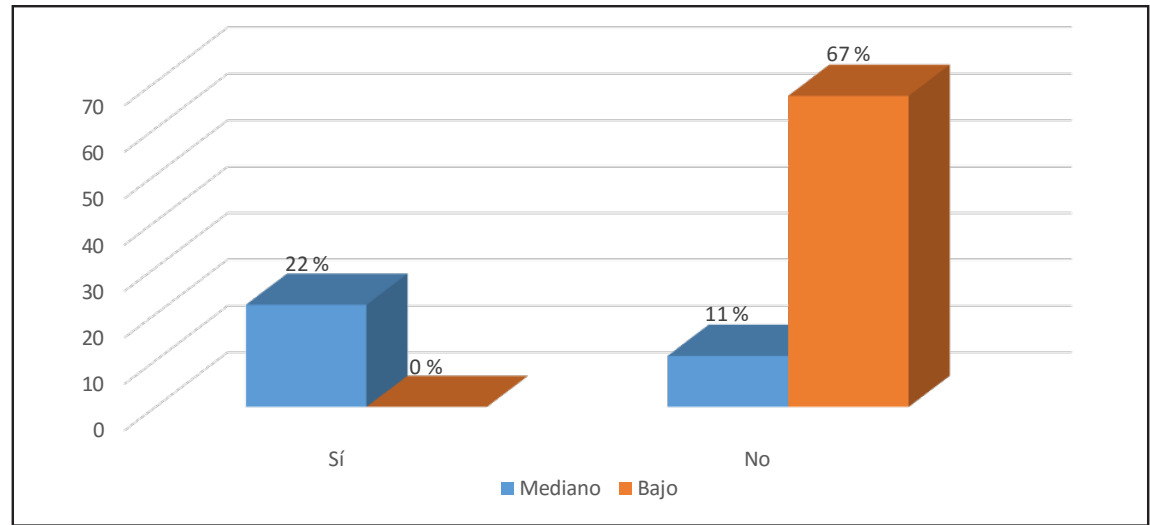

Figura 9: Relación entre el nivel de conocimiento de bioseguridad y la accidentabilidad según la edad de 41-50 años de los trabajadores del CAP.II Chilca-EsSalud-Huancayo 2015.

traumatismos y $17 \%$ por expectoración.

De acuerdo a la figura 4 se muestra que el $67 \%$ de los accidentes laborales no usaban elementos protectores.

La figura 5 muestra que el $33 \%$ no presentaba inmunización activa en el momento del accidente laboral.

De acuerdo a la figura 6 el $67 \%$ de los trabajadores no realizaron acciones inmediatas correctas después del accidente laboral. 
La figura 7 muestra que los pacientes con accidentes laborales creen estar contagiados con hepatitis $\mathrm{B}, \mathrm{VIH}$, TBC y esguinces.

En la figura 8, se presenta que no existe relación significativa entre el nivel de conocimiento $y$ accidentabilidad laboral. Sin embargo observamos que el 3,3 \% de trabajadores con nivel de conocimiento alto no tuvo accidente laboral, mientras que los de nivel de conocimiento mediano y bajo el $20 \%$ si tuvo accidentes laborales. Lo que nos indica que si mejoráramos el nivel de conocimiento de bioseguridad la incidencia de accidentabilidad sería menor.

En la figura 9 se muestra que el $22 \%$ de trabajadores que tuvieron accidentes laborarles con edad entre 41 50 años tuvieron mediano conocimiento $(P<0,05)$.

\section{DISCUSIÓN}

En este estudio se estimó la relación existente entre el Nivel de Conocimientos de Bioseguridad y la Accidentabilidad Laboral en el personal asistencial del CAP II Chica EsSALUD-2015, la misma que de acuerdo a la hipótesis cuando los Niveles de Conocimientos son altos el riesgo de ocurrencia de accidentabilidad es bajo. Razón por la cual, aquellos trabajadores asistenciales con conocimientos altos de Bioseguridad no presentaron ningún accidente laboral, pero en la relación no resulto significativa $(p>0,001)$ toda vez que existen otros factores que influyen en la disminución de los accidentes laborales como son el uso de protectores en forma obligatoria.

En cuanto al uso de Barreras de Protección en Bioseguridad, 20 trabajadores (67 \%) conocen correctamente sobre este tema y 4 (20\%) de ellos tienen accidentes laborales, cifras inferiores a Collado Lilly (2006), donde señala que el $70 \%$ de los profesionales de enfermería tiene conocimientos acerca del uso de barreras protectoras.

En nuestro estudio se reporta que 24 (80 \%) de los trabajadores conocen correctamente acerca de las Medidas de Eliminación de Bioseguridad, cifras iguales a Collado Lilly (2006), que señala que el $80 \%$ de enfermeros tiene conocimientos acerca del adecuado lugar de descarte de material corto punzante, éste valor es semejante al de Tellez Julia, Tovar Maritza (2008), quien señala que un porcentaje de enfermeros no aplica la bioseguridad a la vez, que no hace un buen manejo en cuanto manejo de desechos se refiere.

Según Armas (2007) señala que el $70 \%$ de enfermeros usa siempre guantes, mientras que un $30 \%$ solo a veces, estos resultados difieren de los reportados en nuestro estudio, demostrando que entre quienes conocen la manera correcta del uso de guantes, son el $10 \%$ quienes tampoco tienen accidentes, se concluye que el conocimiento del uso de guantes está asociado a la accidentabilidad laboral, sin embargo no tiene una relación significativa en nuestro estudio, toda vez que es obligatorio el uso de guantes en el establecimiento.

En lo estudiado también se reporta la asociación que existe de riesgo biológico y escala de riesgos relacionado con Conocimientos de Bioseguridad y Accidentabilidad Laboral, evidenciándose asociación significativa $(p<0,05)$

De acuerdo a nuestro estudio, la mayor cantidad de accidentes laborales, que representa un $20 \%$ se produjeron en el establecimiento, también en la investigación de Téllez (2008) se evidencia que existe una importante incidencia de accidentabilidad laboral en la Unidad Quirúrgica, de la misma forma Ramos Sara, et. al (2007) señala que el 65,9\% de trabajadores enfermeros reconocieron haber tenido al menos un accidente laboral.

En nuestro estudio se reportó también, que el mecanismo de trasmisión del accidente laboral fue del tipo percutáneo, representando el $50 \%$, inferior a la de Ramos et al (2007), donde señala de tipo percutáneo en un 56,9\%, semejante a la de Telles y Tovar (2008) quienes indican que la mayoría de accidentes laborales fue por punciones percutáneas, además de cortaduras, e inferior a la de Romero et al (2007) quien informa 436 accidentes laborales entre los años 2005-2007, de ellos, el 81,6\% del tipo percutáneo.

Según nuestro estudio el 33,3 \% de la población creen haber contraído el $\mathrm{VIH}$ al momento del Accidente Laboral, resultando inferior al de Macedo (2006) que señala que el $50 \%$ de las enfermeras no aplica las medidas de Bioseguridad de manera correcta, predisponiendo a los pacientes a una lenta recuperación de su salud y poniéndolo en riesgo de contraer alguna Infección, la infección por VIH influye en cierta forma a un mal manejo en cuento a pacientes se refiere.

Por último el $100 \%$ de los encuestados de nuestra investigación conoce como reportar un accidente laboral por la existencia de formatos que se otorgan en los establecimientos y en el servicio de emergencia de hospitales se rellena en forma obligatoria, resultados no coincidentes con Téllez y Tovar (2008), quienes señalan que no se sigue, en la mayoría de los casos, el protocolo ante accidentes laborales, de la misma forma Romero et al (2009) afirma que existe la necesidad de implementar un programa de prevención y manejo de riesgo laboral, dicho aspecto que debería ser tomado en cuenta para disminuir en cuantía los riesgos laborales. 
Las conclusiones a las cuales se arribaron son:

1. En relación al Nivel de Conocimientos de Bioseguridad y la Accidentabilidad laboral en los trabajadores del CAP II CHILCA Es SALUD 2015 no fue significativa $(p>0,05)$.Sin embargo observamos que el 3,3\% de trabajadores con nivel de conocimiento alto no tuvo accidente laboral, mientras que los de nivel de conocimiento mediano y bajo el $20 \%$ tuvo accidentes laborales. Lo que nos indica que si mejoráramos el nivel de conocimiento de bioseguridad la incidencia de accidentabilidad sería menor.

2. Existe una relación significativa $(p<0,05)$ entre el nivel de conocimientos de bioseguridad y la accidentabilidad laboral según la edad (41-50 años), ocupación en el grupo de vigilantes, limpieza y de enfermeras. Por otro lado según sexo, tiempo de trabajo, estado civil, integrante del comité y última capacitación resultaron no significativos $(p>0,05)$.

3. Existe relación significativa $(p<0,05)$ entre aquellos que no conocen principios de bioseguridad (universalidad) y accidentabilidad laboral con el nivel de conocimiento asimismo también hay una relación significativa $(p<0,05)$ entre aquellos que no conocen principios de bioseguridad (uso de barreras) y accidentabilidad laboral con el nivel de conocimiento.

4. Existe una relación significativa $(p<0,05)$ entre los que tienen conocimientos sobre la aplicación de la escala de riesgos y su relación con la accidentabilidad laboral y el nivel de conocimientos. Se observa que el $12 \%$ de los trabajadores que respondieron en forma incorrecta en relación a la escala de riesgos presentan accidentes laborales y un nivel de conocimiento bajo, también existe relación significativa $(p<0,05)$ entre la respuesta incorrecta sobre el riesgo biológico, la accidentabilidad laboral y el nivel de conocimientos de bioseguridad.

5. Del total de la población estudiada el $20 \%$ presento accidentes laborales, en cuanto a características epidemiológicas de éste suceso, se encontró, que el mecanismo de transmisión predominante fue del tipo percutáneo (50\%), el $67 \%$ no uso un elemento protector al momento del accidente, el $33 \%$ de esta población no estuvo inmunizado, el $67 \%$ actuó de manera incorrecta inmediatamente después del accidente laboral, el $50 \%$ opina de manera incorrecta acerca de cómo comprobar la enfermedad, el 33,3\% cree tener la enfermedad del VIH y el $100 \%$ conoce sobre la forma correcta de reportar el accidente laboral.

\section{REFERENCIAS BIBLIOGRAFÍCAS}

Cordova , M., Mayer , L., \& Horma , F. (2007). Incidencia de Accidentes Laborales en el Personal de Enfermería del Hospital Luis Valverde Vega . Revista Médica: Colegio de Enfermeras de Costa Rica, 2(4), $11-12$.

Galvan , E., \& Gulliano, M. (2006). Influencia De Accidentes Laborales En Relación Con El Servicio De Emergencia. Estudio Retro prospectivo de trabajadores de salud en dicho hospital. Tesis de Maestría, ESSALUD, Lima-Perú.

Gambino, D. (may de 2006). Biosafety in Hospitals. Inside the bibliography revised for a report carried out at the hospital Dr. Salvador. Obtenido de www. bvs.sld.cu/revistas/rst/vol8 1 07/rst10107.html

Jahsi , K. (2006 ). Occupational Safety and Health Administration. Exposición a patógenos transmitidos por la sangre en el trabajo . Washington DC, (EE. UU).

Miranda, R., Montero, M., \& Fernandez, N. (2007). Riesgos Biologicos en la Profesion de la Salud. 6, 3, 25-26.

Paucar, J. (2007). Accidentabilidad Laboral. Estudio prospectivo en enfermeras del Hospital Universitario del Valle. Tesis de Maestria, Santiago-Chile.

Ramos , S., Pino , E., \& Galván , K. (2006). Seroprevalencia De Hepatitis C en el Personal Asistencial del Hospital Regional de Ayacucho y la Microrerd de Salud Huamanga. Estudio Retrospectivo de trabajadores en salud de dicho hospital y Microred. Ayacucho.

Ramos, P. (2007). Salud Ocupacional en Enfermería. Monografía electrónica Bogota.

Romero, A., Cabrera , F., Jimenez , M., \& Ruiz , J. (s.f.). Prevalencia de Accidentes Biológicos Por Punción Accidental en el Hospital Virgen de La Victoria. Estudio Retrospectivo Exploratorio de accidentes declarados de dicho hospital. Tesis de Licenciado, Hospital Virgen de La Victoria, Lambayeque- Perú.

Sedano, G. (sep de 2007). Consecuencias de los Accidentes de Trabajo. Obtenido de www.anec. org.co

Telles , J. (2008). Medidas de Bioseguridad y Accidentabilidad Laboral. Estudio prospectivo en enfermeras del Hospital José María Vargas. Tesis de Licenciado, Universidad Central de Venezuela, Caracas-Venezuela. 